\title{
Experiment and modeling into drilling of micro-hole on TC4 by electrochemical jet machining
}

\author{
Dongxiao Song ${ }^{1, *}$, Liangcai Xiong ${ }^{1}$, Yuhong Long ${ }^{2}$, and Tielin Shi ${ }^{1}$ \\ ${ }^{1}$ School of Mechanical Science \& Engineering, Huazhong University of Science and Technology, \\ Wuhan, 430074, China \\ ${ }^{2}$ School of Mechanical and Electrical Engineering, Guilin University Of Electronic Technology, Guilin, \\ 541010, China
}

Keywords: electrochemical jet machining, TC4, micro-hole, response surface analysis, profile prediction

\begin{abstract}
This paper studied the rule of micro-hole in electrochemical jet machining (EJM) of TC4 alloy and established the mathematical model of machining process and predicted the machining profile. Considering the influence of machining gap and machining time, orthogonal experiment was designed. This paper established the mathematical model of the electrochemical jet machining process of TC4 alloy based on the response surface analysis (RSA) method. The results indicate that the electrochemical jet can improve the directivity of machining, reducing the machining gap can improve the machining efficiency, but the jet will cause secondary corrosion and abrupt change of current at the edge of inlet. The mathematical model based on response surface analysis is accurate after variance test. The experimental results show that the average error between the established prediction model of machining depth and the actual value is $2.32 \%$, and the average error of the prediction model of inlet radius is $2.18 \%$.
\end{abstract}

\section{Introduction}

Electrochemical Jet Machining (EJM) is a machining technology based on anodic dissolution. EJM can make the electric field distribution more concentrated compared with traditional electrochemical machining, it allows for more micro processing [1,2]. Moreover, the anode tool has no loss, and the wear of tool material can be avoided compare with traditional cutting process, which is very suitable for the processing of difficult-machining metal materials such as titanium alloy. Electrochemical machining (ECM) has almost no recasting layer compared with laser ablation, heat-affected zone and other regions that have serious influence on the material $[3,4,5,6]$.

\footnotetext{
* Corresponding author: songdongxiao@hust.edu.cn
} 
At present, scholars have done much work on the corrosion characteristics, machining methods and finite element model establishment of materials in the EJM process [7,8,9,10], but for the specific machining process, the mathematical model between each parameter and machining profile is less studied. The establishment of multi-input and multi-output model between processing parameters and processing results can predict the processing results and design the process plan according to the constraint conditions, to improve the production efficiency, which has a higher guiding significance for the actual production. Jansel et al. modeled EDM processing by means of soft computing [11]. Asokan et al. used artificial neural networks to model the electrochemical machining process [12]. Munda et al. used response surface analysis to model the electrochemical machining process of stainless steel [13]. All these works have proved that parametric modeling method has a very high value for the optimization of machining process. However, there are few reports on the establishment of parametric models for electrochemical jet machining.

In this paper, we analyzed the influence of machining parameters on the profile of micro-hole on the surface of TC4 alloy through orthogonal experiment and studied the influence rule of each parameter on the profile of micro-hole. And established the mathematical model between the jet electrochemical machining parameters and the profile of micro-hole based on the response surface analysis method to realize the prediction of the micro-hole profile and provide guidance for the processing technology.

\section{Experimental setup}

Material and equipment. Cold-rolled TC4 titanium alloy plate with thickness of $5 \mathrm{~mm}$ was selected as the experimental material. The sample size of $30 \mathrm{~mm} \times 30 \mathrm{~mm}$ is cut by a plate cutting machine. Table 1 shows the chemical composition.

Table 1. Chemical composition of Titanium alloy sheet.

\begin{tabular}{ccccccccc}
\hline Elements & $\mathrm{Fe}$ & $\mathrm{C}$ & $\mathrm{N}$ & $\mathrm{H}$ & $\mathrm{O}$ & $\mathrm{Al}$ & $\mathrm{V}$ & $\mathrm{Ti}$ \\
\hline Proportion (\%) & 0.2 & 0.05 & 0.03 & 0.005 & 0.1 & 6.1 & 4.0 & $\begin{array}{c}\mathrm{Bal} \\
.\end{array}$ \\
\hline
\end{tabular}

We use electrochemical jet machining system. The repeated positioning accuracy of XYZ triaxial displacement platform is less than $0.02 \mathrm{~mm}$. The nozzle is a $16 \mathrm{G}$ point glue nozzle with an inner diameter of $1.2 \mathrm{~mm}$ and an outer diameter of $1.6 \mathrm{~mm}$. The outer wall is insulated by a heat-shrinkable tube, and the diameter of the nozzle is $2.3 \mathrm{~mm}$ after the insulation treatment. The electrolyte is $20 \% \mathrm{NaCl}$ solution, the jet velocity is $1.8 \mathrm{~m} / \mathrm{s}$, and the processing voltage is $36 \mathrm{~V}$.

NI USB-6251 acquisition card was used to collect the processing current data at a frequency of $100 \mathrm{~Hz}$ during machining processing. After finishing the processing, the surface profile of TC4 titanium alloy was measured using the optical 3D surface metrology equipment and the surface $3 \mathrm{D}$ point cloud data was obtained. The point cloud data were further processed with GOM Inspect 2019 software to measure the section size of the machining position. Fig. 1(a) shows the point cloud data imported into GOM Inspect 2019 and make a section along the black line in the figure. Fig. 1(b) shows the selected machining profile parameters: micro hole depth(D), radius at the inlet of micro hole(R0), radius at the $1 / 4$ depth of surface of micro hole(R1), radius at the $1 / 2$ depth of surface(R2), radius at the $3 / 4$ depth of surface(R3). We define the inlet radius is $\mathrm{R} 0$; the average inner radius is $(\mathrm{R} 1+\mathrm{R} 2+\mathrm{R} 3) / 3$; the upper taper is $4(\mathrm{R} 1-\mathrm{R} 2) / \mathrm{D}$; the lower taper is $4(\mathrm{R} 2-\mathrm{R} 3) / \mathrm{D}$; the average taper is $4(\mathrm{R} 1-\mathrm{R} 3) / \mathrm{D}$; the depth-diameter ratio is $\mathrm{D} / \mathrm{R} 0$. 


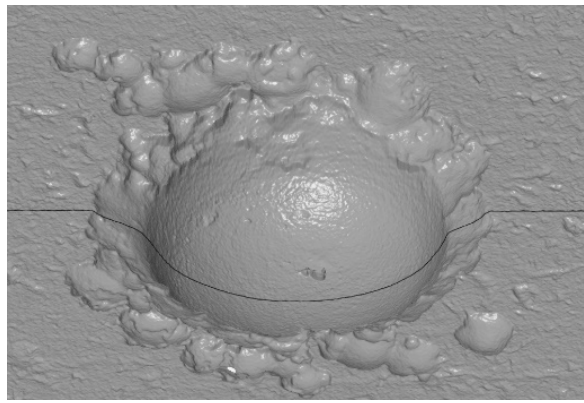

(a) Measured data

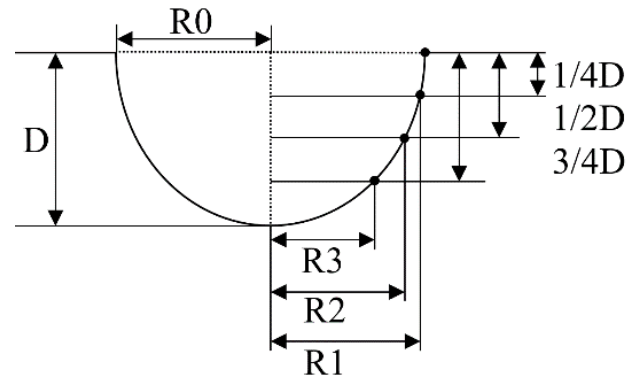

(b) Parameter definition

Fig. 1. Measurement profile and parameter definition.

Experient design. To study the influence of the gap between the nozzle and the workpiece and the processing time on the surface profile of TC4 titanium alloy in the process of EJM, we designed the following orthogonal experiment. Three processing gaps were selected: $0.3 \mathrm{~mm}, 0.5 \mathrm{~mm}$ and $0.7 \mathrm{~mm}$; and four processing time: $1 \mathrm{~min}, 2 \mathrm{~min}, 3 \mathrm{~min}$ and $5 \mathrm{~min}$.

To establish the mathematical model of the machining process, the response surface analysis (RSA) method is used to predict the machining profile under the condition of given process parameters. Through the experiment of two factors and three levels, the machining gap and machining time are independent variables, and the micro-hole depth and inlet radius are response values.

\section{Analysis of machining profile}

Fig. 2 shows the cross-section profile of micro-holes with different machining gap. With the decrease of the machining gap, the processing amount in the depth direction of the micro-hole increases from Fig. 2(a) (d), while the processing amount in the diameter direction increases relatively less. The processing of deeper micro holes can be realized by reducing the machining gap. By comparing Fig. 2(a) and Fig. 2(b) (d), secondary corrosion occurred at the edge during 2-5min machining at the inlet edge of the micro-hole, leading to larger inlet taper. The secondary corrosion at the inlet edge is caused by the fluid in the central part flowing to the side wall of the micro-hole under the pressure of the upper jet after the jet impinges on the tiny hole and flows out along the side wall. As the jet velocity is high, the solution will reflect out from the micro-hole and contact with the bottom surface of the machining nozzle, making the bottom of the nozzle conductive with the workpiece, leading to the secondary corrosion of the workpiece.

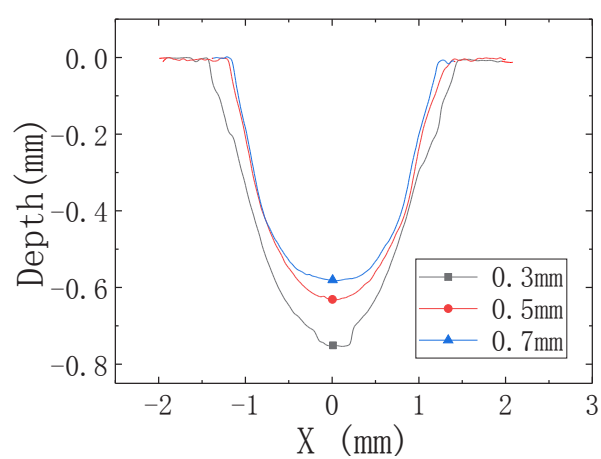

(a) Machining $3 \mathrm{~min}$

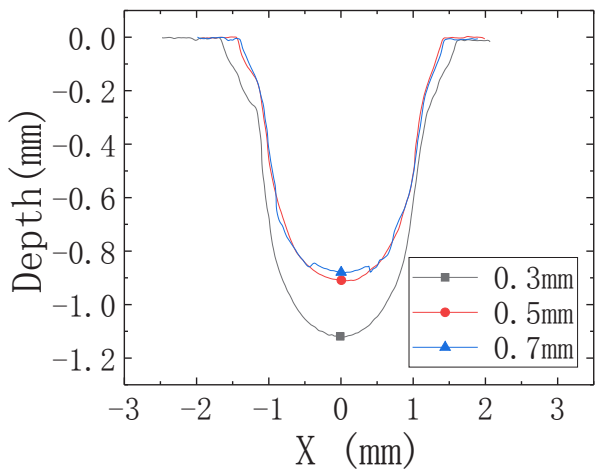

(b) Machining $5 \mathrm{~min}$ 


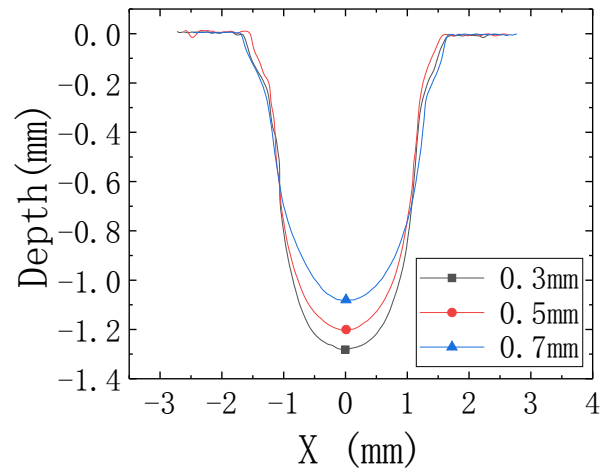

(c) Machining $3 \mathrm{~min}$

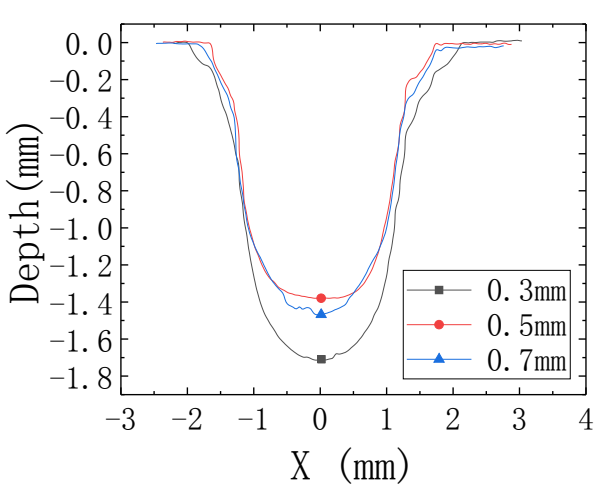

(d) Machining $5 \mathrm{~min}$

Fig. 2. The cross-section profile of micropores with different gap.

Fig. 3(a) is changes in the taper of the upper and lower parts, the upper part of the inner wall is the average taper from the surface of the micro hole to $1 / 2$ depth, and the lower part of the inner wall is the average taper from $1 / 2$ depth to the bottom of the micro hole. The upper taper of the micro-hole is always smaller than the average taper of the bottom at any processing time under the same gap from Fig. 3(a). With the increase of the machining time, the taper of the upper and lower parts decreased significantly, and the taper decreased most significantly at the machining time of $2 \mathrm{~min}$. At the processing time of $5 \mathrm{~min}$, the taper is close to 1 for all three processing gaps. Fig. 3(b) is change in average taper, with the increase of machining time, the average taper decreases, which proves the feasibility of electrochemical machining by jet for machining small holes with large depth-to-diameter ratio. The taper of the micro-holes decreases due to the decrease of jet velocity and the deterioration of jet orientation with the increase of the machining clearance.

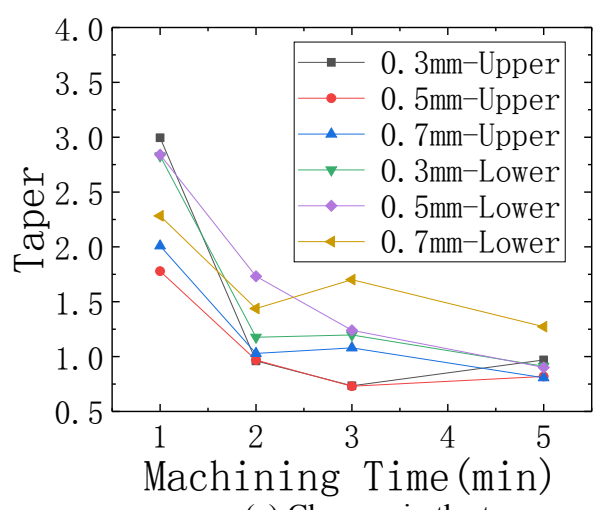

(a) Changes in the taper

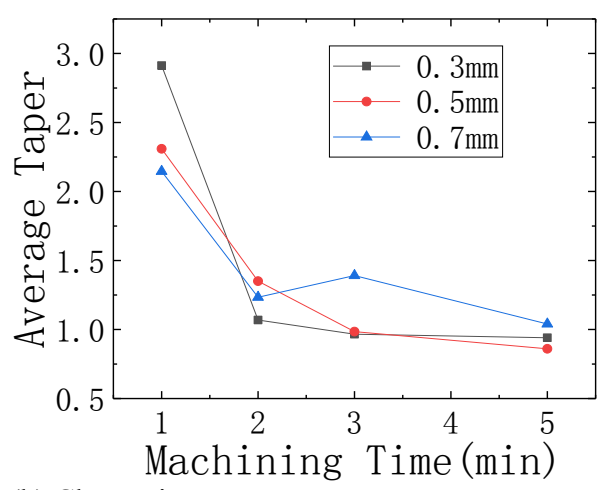

(b) Change in average taper

Fig. 3. The taper of inner wall varies with process parameters. 


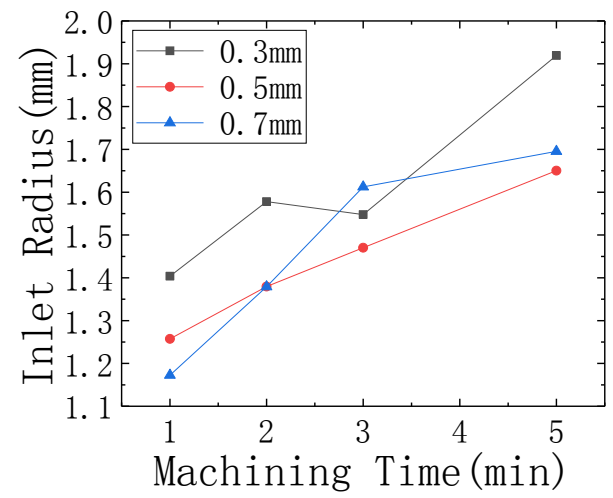

(a) the inlet radius changes with time

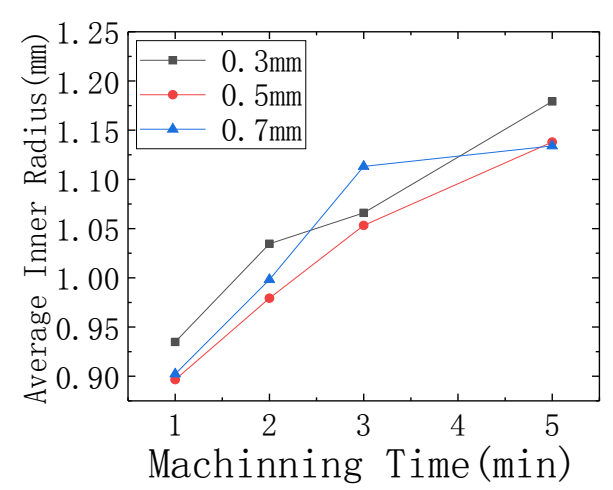

(b) the average inner radius changes with time

Fig. 4. Inlet radius and average inner radius.

Fig. 4(a) we found that the gap reduction will result in a larger inlet radius, due to secondary corrosion caused by the electrolyte reflected from the tiny hole in contact with the bottom surface of the nozzle, which enlarges the inlet radius. In order to solve this problem, after processing for a period of time with a higher processing clearance, the processing clearance can be reduced again and the nozzle can be sunk into the tiny hole to reduce the secondary corrosion at the entrance. It can be seen from Fig. 4(b), the influence of different machining clearance on the average inner diameter of micro hole is less than the inlet radius. After machining $5 \mathrm{~min}$ at the gap of $0.3 \mathrm{~mm}$, the average inner radius was $1.1792 \mathrm{~mm}$, still smaller than the inside diameter of $1.2 \mathrm{~mm}$ and the outside diameter of $1.6 \mathrm{~mm}$ of the nozzle, which proved that the electrochemical machining of jet flow has a high directivity and can realize the machining of finer structures on the workpiece than the tool.

Fig. 5(a) and (b) respectively show the variation of micro hole depth and depth-diameter ratio with processing time. From Fig. 5(a), depth increases linearly with processing time, However, as shown in Fig. 5(b), the depth-diameter ratio increases rapidly in the processing of 1-3min but slows down in the processing of $5 \mathrm{~min}$. The increase of depth-diameter ratio is slow due to the increase of secondary corrosion in inlet radius with the extension of processing time.
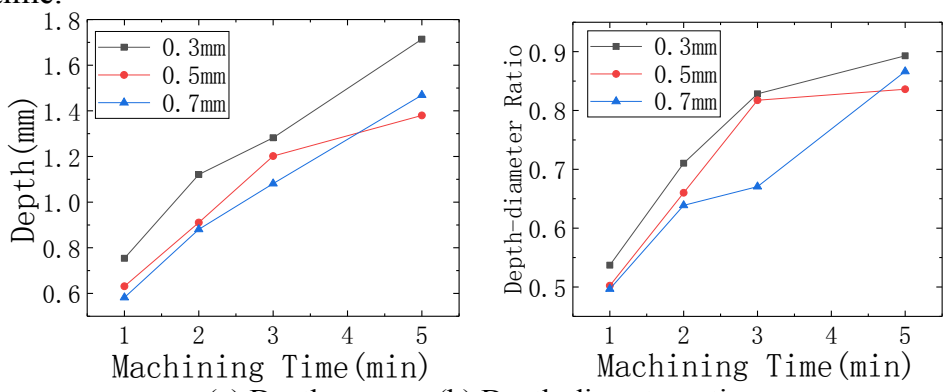

(a) Depth

(b) Depth-diameter ratio

Fig. 5. Depth and depth-diameter ratio.

\section{Establishment and verification of machining model}

The response surface method simulates the real limit state surface through a series of deterministic 'experiments' fitting into a response surface [14]. The basic idea is to assume that an analytic expression between the limit state function and the basic variables, including 
some unknown parameters, replaces the actual structural limit state function [15], which cannot be explicitly expressed.

Response surface modeling. The quadratic model is used to machining deep. The F-value of model is 37.58 , indicating that the model is significant and the probability of errors due to noise is less than $0.66 \%$. The measured variance $\mathrm{R}^{2}=0.9843$ is close to 1 (the closer $R^{2}$ is to 1 , the more significant it is), and $R^{2}$ pred signal-to-noise ratio $A_{p}$ is 16.2837 (greater than 4 , the model is satisfactory). The predicted fitting coefficient $\mathrm{R}_{\text {pred }}{ }^{2}=0.8439$ is close to the modified fitting coefficient $\mathrm{R}_{\text {adj }}^{2}=0.9581$ (the closer the two values are, the more significant the model is). The final machining deep mathematical model is Eq. 1.

$$
\mathrm{D}=0.926-2.273 \mathrm{G}+0.389 \mathrm{~T}-0.046 \mathrm{GT}+1.896 \mathrm{G}^{2}-0.025 \mathrm{~T}^{2}
$$

A custom model was used for machining radius. The F-value of model is 18.2 , indicating that the model is significant and the probability of errors due to noise is less than $0.4 \%$. The measured variance $\mathrm{R}^{2}=0.9161$ is close to 1 , and $\mathrm{R}_{\text {pred }}^{2}$ signal-to-noise ratio $\mathrm{A}_{\mathrm{p}}$ is 11.4311 . The predicted fitting coefficient $\mathrm{R}_{\text {pred }}^{2}=0.7475$ is close to the modified fitting coefficient $\mathrm{R}_{\text {adj }}^{2}=0.8657$. The final machining radius mathematical model is Eq. 2.

$$
\mathrm{R}=1.883-2.802 \mathrm{G}+0.119 \mathrm{~T}+2.477 \mathrm{G}^{2}
$$

Model validation. Fig. 6 respectively shows the relationship between the actual value and the predicted value of machining depth and inlet radius. It can be seen from the figure that the deviation between the predicted value and the actual value is distributed along the diagonal, indicating that the predicted value through the model is very close to the actual value, and the established model is more accurate.

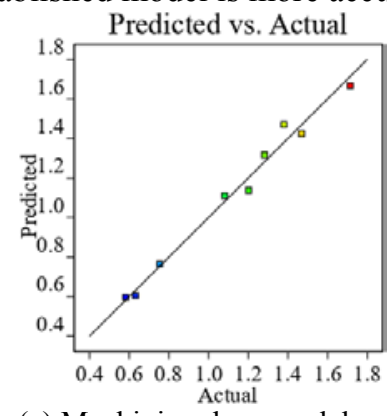

(a) Machining deep model

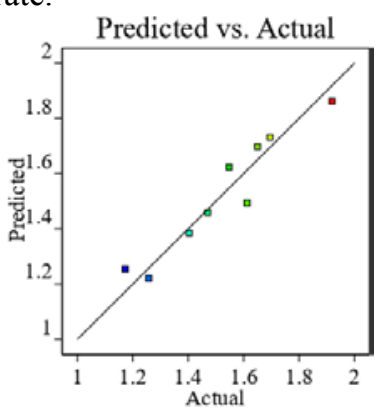

(b) Machining radius model

Fig. 6. The predicted values of the two models were compared with the actual values.

Fig. 7(a) shows the response surface of machining clearance and machining time to machining depth. It can be seen from the figure that reducing machining clearance can improve machining depth. Fig. 7(b) shows the response surface of the machining clearance and machining time to the inlet radius. It can be seen from the figure that when the machining clearance is $0.3 \sim 0.7 \mathrm{~mm}$, the inlet radius increases first and then decreases with the increase of the machining clearance. This is due to the reduction of machining gap, the bottom of the nozzle is close to the workpiece, causing secondary corrosion at the entrance; When the machining clearance increases, the directivity of jet becomes worse, leading to the increase of inlet radius. 


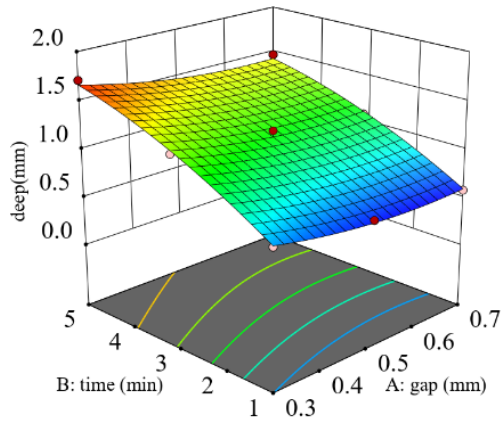

(a) Machining deep model

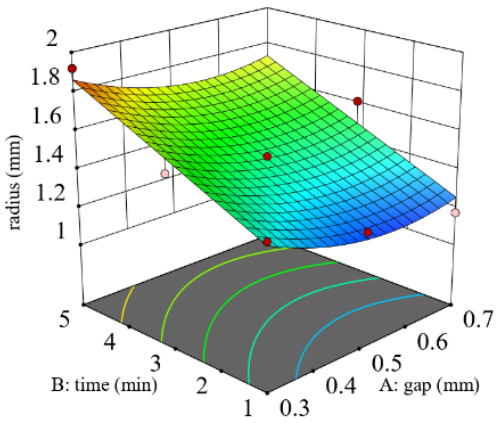

(b) Machining radius model

Fig. 7. The response surface figure of machining deep model and machining radius model.

Table 2 shows the verification results of the mathematical models, compares the morphs of three different gaps processed for $2 \mathrm{~min}$. By comparing the actual measured values with the predicted values of the model, the average error of the prediction model established in this paper for machining depth is $2.32 \%$, the average error of the prediction model for inlet radius is $2.18 \%$, and the total average error of the two models is $2.25 \%$ from Table 6 .

Table 2. Verify experimental results.

\begin{tabular}{|c|c|c|c|c|c|c|c|c|}
\hline \multirow{2}{*}{ No. } & \multirow{2}{*}{$\begin{array}{c}\text { Gap } \\
(\mathrm{mm})\end{array}$} & \multirow{2}{*}{$\begin{array}{l}\text { Time } \\
(\mathrm{min})\end{array}$} & \multicolumn{3}{|c|}{ Deep } & \multicolumn{3}{|c|}{ Radius } \\
\hline & & & Act. & Pred. & Err. & Act. & Pred. & Err. \\
\hline 1 & 0.3 & 2 & 1.1208 & 1.0661 & 0.0513 & 1.5780 & 1.5164 & 0.0406 \\
\hline 2 & 0.5 & 2 & 0.9107 & 0.8965 & 0.0158 & 1.3796 & 1.3514 & 0.0208 \\
\hline 3 & 0.7 & 2 & 0.8808 & 0.8786 & 0.0025 & 1.3790 & 1.3846 & 0.0040 \\
\hline
\end{tabular}

There is a certain error between the predicted value of the model and the actual measured value, but the error is relatively small and within the allowable range. Therefore, it can be proved that the mathematical model established by response surface analysis method is feasible for modeling the electrochemical machining process of jet. The processing results can be predicted efficiently by using this model, and the process parameters can be further optimized, and the optimal processing route can be calculated by setting constraints under the given profile.

\section{Summary}

In this paper, through experimental analysis of TC4 electrochemical machining micro-hole morphology, machining clearance and machining time between the change rule, using response surface analysis method to model the machining parameters and machining morphology, the following conclusions are found:

Under the same processing time, with the reduction of the processing clearance, the processing amount in the depth direction of the micro hole increases, while the processing amount in the diameter direction increases relatively less. At the entrance edge of the micro hole, secondary corrosion occurred at the edge during 2-5min machining, leading to larger entrance taper. With the extension of processing time, the average taper decreases.

Based on response surface analysis, a mathematical model between machining clearance $\mathrm{G}$, machining time $\mathrm{T}$ and machining depth $\mathrm{D}$ is established. It is verified that the prediction error of the model is $2.32 \%$. The mathematical model between machining clearance $\mathrm{G}$, machining time $\mathrm{T}$ and inlet radius $\mathrm{R}$ is established. It is verified that the prediction error of the model is $2.18 \%$. 
The Scientific Research Program is supported by Hubei Provincial Natural Science Foundation of China (Program No. 2017CFB658) and the National Natural Science Foundation of China (Grant No. 51975192).

\section{References}

1. Pajak P, Desilva A, Harrison D. Precision and efficiency of laser assisted jet electrochemical machining. Precis Eng, 2006, 30(3): 288-298.

2. Pajak P, Desilva A, McGeough J. Modelling the aspects of precision and efficiency in laser-assisted jet electrochemical machining (LAJECM). J Mater Process Tech, 2004, 149(1-3): 512-518.

3. Desilva A, Pajak P, McGeough J. Thermal effects in laser assisted jet electrochemical machining. CIRP Ann Manuf Technol, 2011, 60(1): 243-246.

4. Hackert M, Paul R, Martin A. Study on the dynamic generation of the jet shape in Jet Electrochemical Machining. J Mater Process Tech, 2015, 223: 240-251.

5. Schubert A, Hackert M, Martin A. Generation of Complex Surfaces by Superimposed Multi-dimensional Motion in Electrochemical Machining. Procedia CIRP, 2016, 42: 384-389.

6. Liu W, Zhang H, Luo Z. Electrochemical micromachining on titanium using the NaCl-containing ethylene glycol electrolyte. J Mater Process Tech, 2018, 255: 784-794.

7. Yudi W, Zhengyang X, An Z. Electrochemical dissolution behavior of Ti-45Al-2Mn-2Nb+0.8 vol\% TiB2 XD alloy in $\mathrm{NaCl}$ and $\mathrm{NaNO} 3$ solutions. Corros Sci, 2019, 157: 357-369.

8. Jinxing L, Xiaolong F, Di Z. Jet electrochemical machining of multi-grooves by using tube electrodes in a row. J Mater Process Tech, 2020, 283: 116705.

9. Qingfeng, Y., Pengyue, W., Zhiqiang, Q. et al. Electrical discharge drilling assisted with bubbles produced by electrochemical reaction. Int J Adv Manuf Technol 109, 919-928 (2020).

10. Minghao, L., Yong, L., Siying, L. et al. Theoretical and experimental study on micro ultrasonic-assisted electrochemical drilling with high speed electrode. Int J Adv Manuf Technol 107, 815-826 (2020).

11. Leyva-Bravo, J., Chiñas-Sanchez, P., Hernandez-Rodriguez, A. et al. Electrochemical discharge machining modeling through different soft computing approaches. Int $\mathrm{J}$ Adv Manuf Technol 106, 3587-3596 (2020).

12. Asokan, P., Ravi Kumar, R., Jeyapaul, R. et al. Development of multi-objective optimization models for electrochemical machining process. Int J Adv Manuf Technol 39, 55-63 (2008).

13. Munda, J., Bhattacharyya, B. Investigation into electrochemical micromachining (EMM) through response surface methodology based approach. Int J Adv Manuf Technol 35, 821-832 (2008).

14. Qingfeng, Y., Pengyue, W., Zhiqiang, Q. et al. Electrical discharge drilling assisted with bubbles produced by electrochemical reaction. Int J Adv Manuf Technol 109, 919-928 (2020).

15. Minghao, L., Yong, L., Siying, L. et al. Theoretical and experimental study on micro ultrasonic-assisted electrochemical drilling with high speed electrode. Int J Adv Manuf Technol 107, 815-826 (2020). 